\title{
A new method for quantitative and qualitative representation of the noises type in Allan (and related) variances
}

\author{
Kamil Maciuk ${ }^{1 *}$ [D, Jacek Kudrys ${ }^{1}$, Mohammad Bagherbandi ${ }^{2,3}$ and Igor V. Bezmenov ${ }^{4}$
}

\begin{abstract}
Allan variances and its related methods are commonly used to analyse a sequence of data in the time domain to measure frequency stability of oscillators. It allows for a determination of noise type as a function of the averaging time. This method is one of the most popular for identifying different noise types in the sensors data, and it is usually used for a wide range of studies on the stability of oscillators or atomic clocks. In this paper, the authors propose their own algorithm for a qualitative and quantitative expression of noise type using Allan and related variation. The methods currently used allow for the determination of the noise type graphically only, without quantifying the type and percentages for each of the integration steps. Our study allows for a numerical interpretation of the Allan (and related) variances data. A numerical example of the described method is based on GPS satellite clock corrections for 1825-2086 GPS Week (5 years) based on daily 30-s sampling interval products.
\end{abstract}

Keywords: Allan deviation, Clock analysis, GPS, GNSS, Noises

\section{Introduction}

Allan deviation (ADEV) is a root of Allan variance (AVAR) and was established in the 1980s (Allan 1983, 1987) as a time-domain method for characterisation of the performance of atomic clocks and oscillators. It allows-in a simple way-to express the time-domain stability of the frequency source by means of $\sigma(\tau)-\tau$ plots, where $\sigma(\tau)$ is an Allan deviation for $\tau$ and $\tau$ is an averaging time (Fig. 1), which measures frequency stability versus time over which the frequency is averaged (Riley 2008).

Allan (and related) variance analysis is a very important and effective tool that helps to investigate and study the characteristics of the errors/noises in the GNSS observation for some precise positioning applications. There are three important parameters and quantities in the atomic clocks, namely the uncertainty (or accuracy),

\footnotetext{
*Correspondence: maciuk@agh.edu.pl

${ }^{1}$ Faculty of Mining Surveying and Environmental Engineering, AGH

University of Science and Technology, Krakow, Poland

Full list of author information is available at the end of the article
}

instability (statistical uncertainty) and reproducibility that are important for the clock-based geodesy applications (McGrew et al. 2018). Therefore, we will be able to build precise error models using this type of investigation using GNSS data.

Currently, in the research in the field of stability of atomic clocks and oscillators, the adjustment of the type of noise to the angle of the graph is done manually based on graphic fitting (Amiri-Simkooei et al. 2007; Senior et al. 2008; Shi et al. 2019). In this paper, authors describe their own algorithm for quantitative and qualitative description of noise type in a time series based on the daily clock products with 30-s sampling interval for a 5-year period and including one year (1 January-31 December 2018, 1982-2034 GPS Week) in detailed description (raw data-Fig. 3, MDEV and method visualisation-Fig. 4).

\section{Algorithm description}

The calculated values of Allan and related deviation for adopted averaging times usually expressed as octave or decade intervals characterise the noise level of the tested 


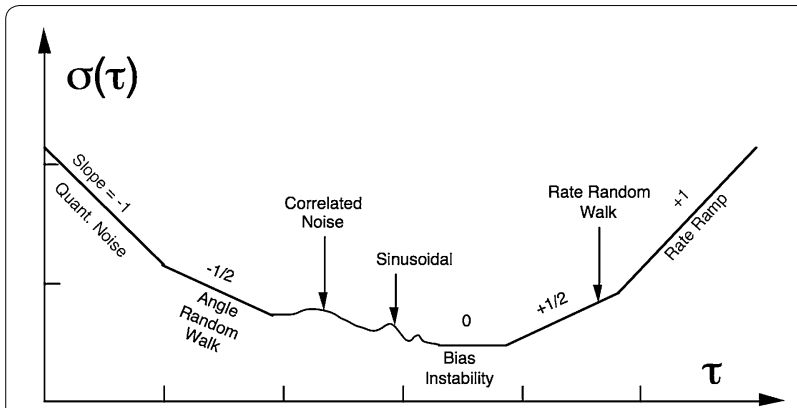

Fig. 1 Allan variance plot for typical data analysis. The cluster time of length $\tau$ could take different units in time, e.g. microseconds, seconds, minutes or hours as well as $\sigma(\tau)$, i.e. angular velocity, e.g. rad/s or o/h for gyros, depending on sensor type (Hidalgo et al. 2012)

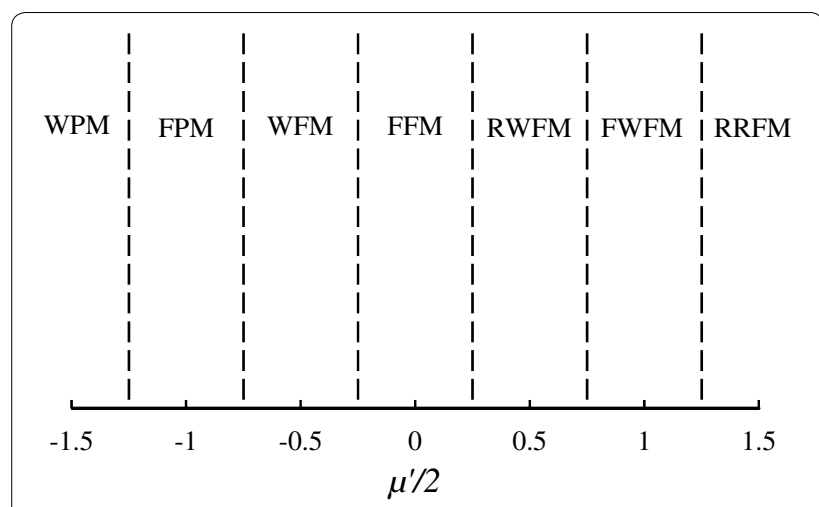

Fig. $2 \mu^{\prime} / 2$ coefficient ranges for each noise type (WPM — White PM \{Phase Modulation\}, FPM-Flicker PM, WFM-White FM \{Frequency Modulation\}, FFM — Flicker FM, RWFM — Random Walk FM, FWFMFlicker Walk FM, RRFM—Random Run FM)

signal. An analysis dominant noise is identified for each $\tau$ using the power-law spectrum index, based on the values of ADEV, allowing for a graphical interpretation of the noise type. This method allows for an assignment noise type for each value of ADEV and $\tau$ based on:

$$
\frac{\mu^{\prime}}{2}=\frac{\partial(\ln \sigma)}{\partial(\ln \tau)}
$$

where the numerator is a difference of two consecutive logarithms of Allan deviation $(\sigma)$ and the denominator is a difference between two consecutive logarithms of averaging times $\tau$, which corresponds to values from the numerator. Depending on the $\mu^{\prime} / 2$ value, the noise character is classified as:

Calculated $\mu^{\prime} / 2$ values using Eq. (1) need to be assigned to each range as half the distance between the individual values; for $\mathrm{WPM}<-1.25$, for $-1.25 \leq \mathrm{FPM}<0.75$, etc. (Fig. 2).

\section{Case study}

Phase data raw GPS clock corrections of three selected satellites/block/clock type: G03/2F/Rb, G11/2R/Rb, G24/2F/Cs obtained from the IGS-MGEX (International GNSS Service The Multi-GNSS Experiment) project were subjected to pre-processing to remove outliers and discontinuities (Fig. 3) using Median Absolute Deviation (MAD) value (Riley 2008). Below figure shows phase values (clock corrections) of 3 GPS satellite clocks for the whole 2018 year.

Based on the above pre-processed data, the MDEV (Modified Allan deviation) was calculated. Figure 4 top presents MDEV for each analysed satellite with added noises type (dashed line) for each part of the chart. For example, satellite G24 is represented by the FPM for $30-60 \mathrm{~s} \sigma$, WFM for $60-1966080 \mathrm{~s} \sigma$, and RWMF for the rest of $\sigma$ values. The noise type when using MDEV (in this example) is uniquely determined by the value of $\mu^{\prime} / 2$ which depends on the averaging interval. The clock of all GNSS satellites usually contains noise of all types listed in the article. Differences exist in the averaging parameter intervals at which a particular type of noise appears (Senior et al. 2008; Heo et al. 2010; Griggs et al. 2014).
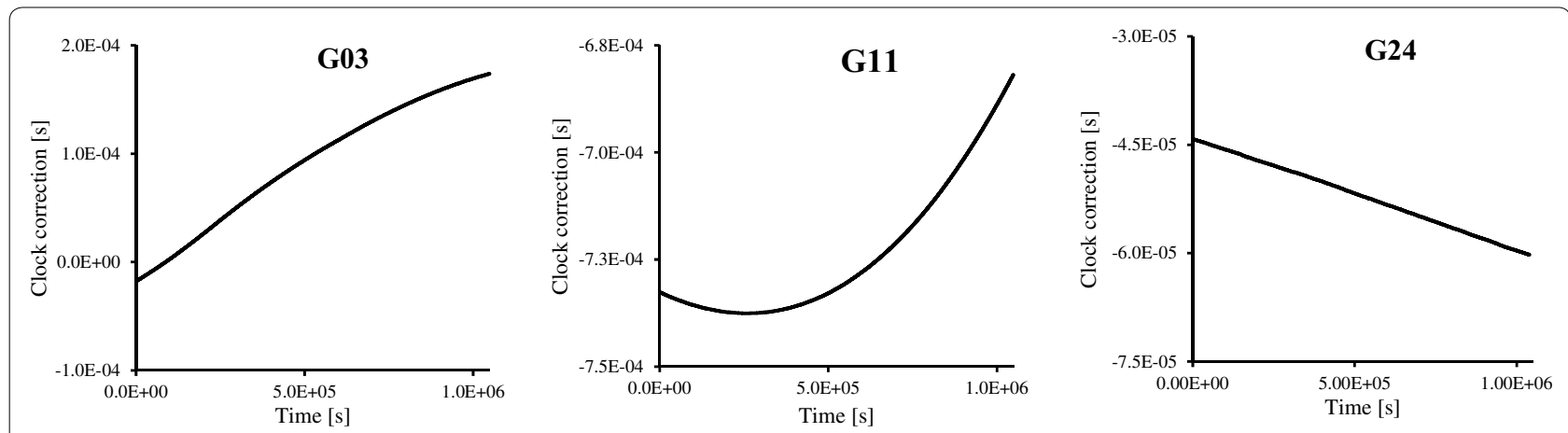

Fig. 3 Cleaned phase data of the analysed satellites clock corrections (1982-2034 GPS Week) 

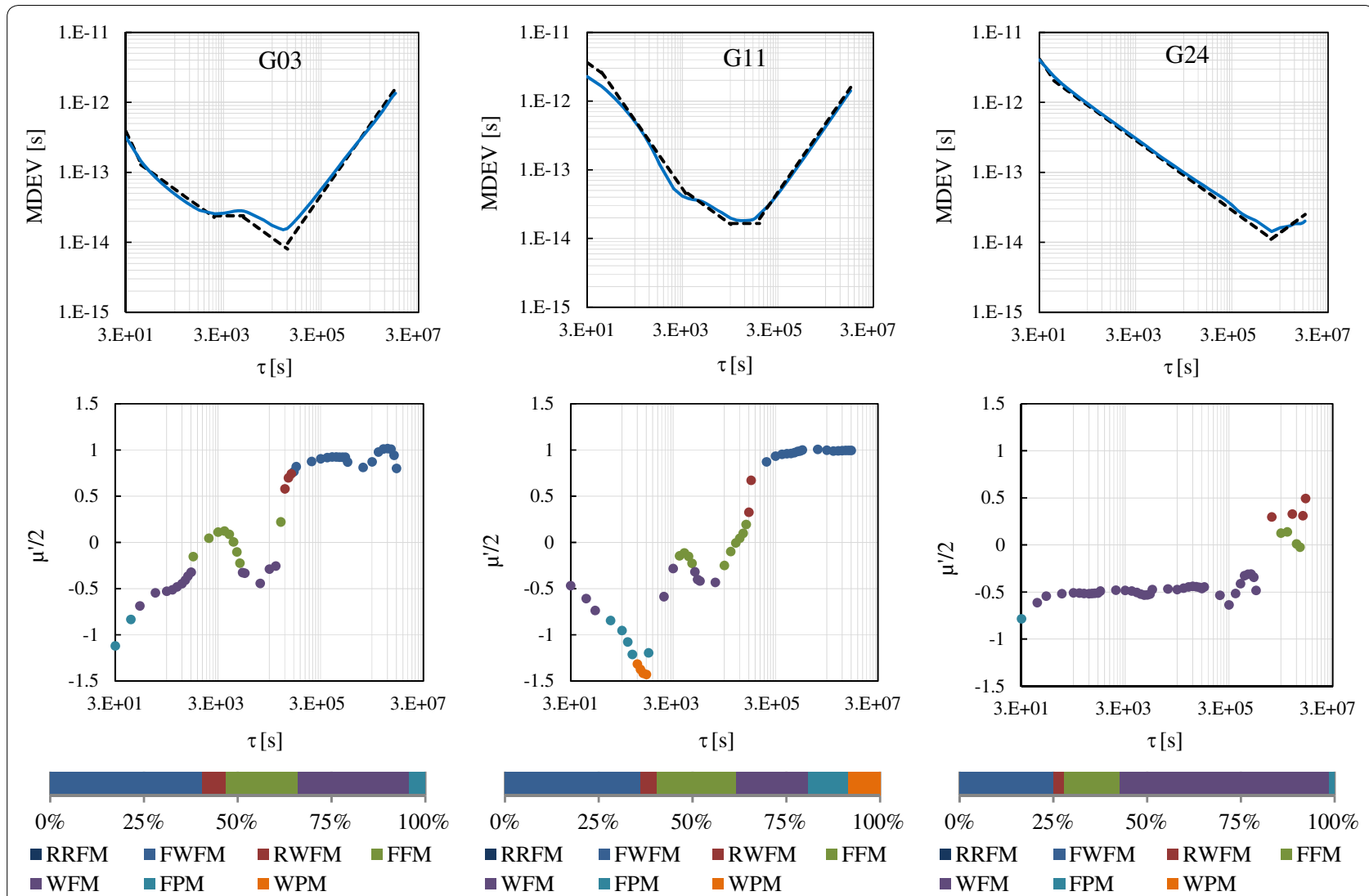

Fig. 4 The noise processes in MDEV variance plot with fitted noises slopes (top), proposed method (middle) and percentage distribution of noise types of the analysed data based on the proposed method (bottom)

Figure 4 middle part shows representation of noise types determined by the authors' method distinguished by noise type, which shows in a different, more accessible way charts used for presenting Allan variance. Bottom part of this figure shows the percentage distribution of each noise type for all the analysed time series-G03, G11 and G24, respectively-based on a simple numerical calculations. This method allows for an assigning of noise

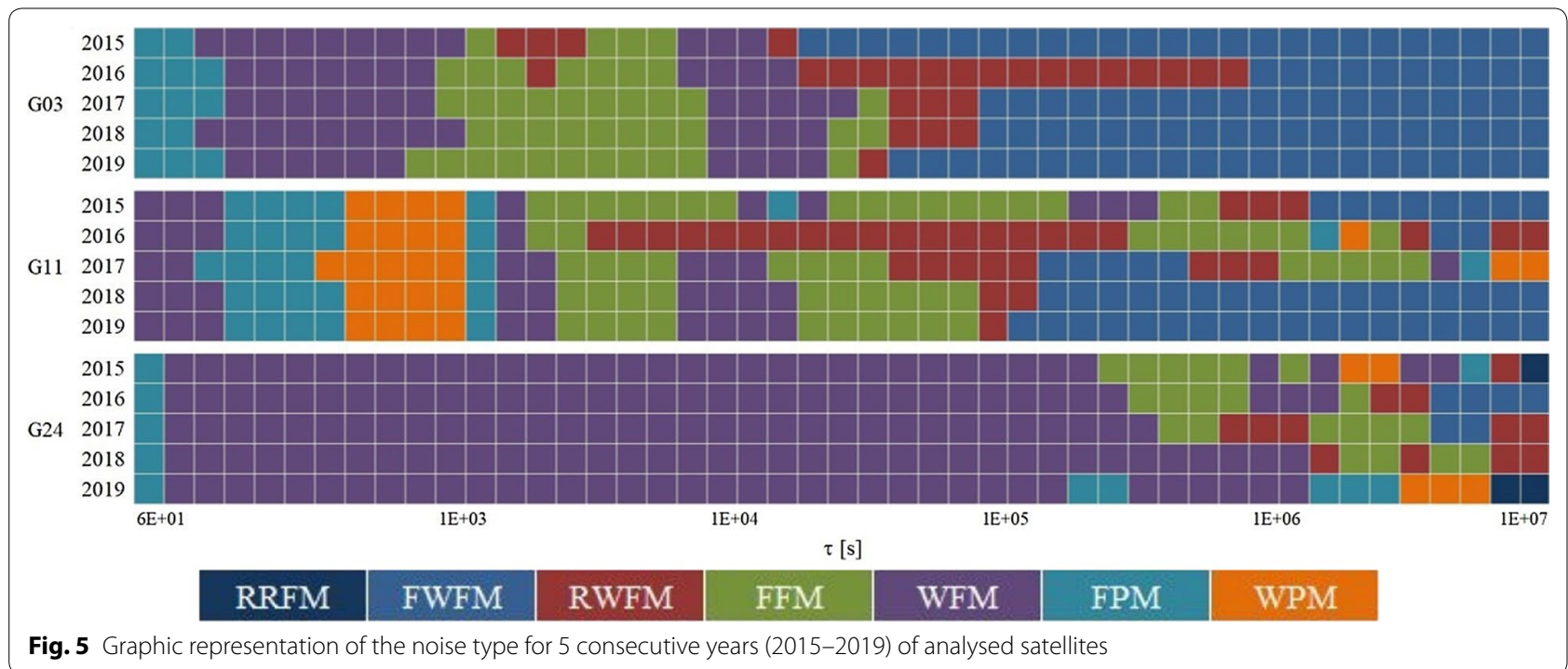


types and their percentage distribution numerically. We cannot gain more by more averaging when the flicker frequency region is reached. Because, the noise type will become random walk by averaging more over longer time span. Therefore, continuing to average will degrade the stability of the clock (Lombardi 2016).

Figure 5 shows a representation of the data on Fig. 4 (middle) for 5 consecutive years (2015-2019) which in much more better way allows for (a) interpretation of the noise type for each averaging time $(\tau)$ and (b) comparison of repeatability and stability of the analysed date for a selected period of time. Below figure shows analysis of the noise types for GPS onboard clocks in annual terms.

Comparison between years for each of the clock reveals great repeatability of the satellite G24. In case of other satellites, there are similar behaviour of all years instead of 2016 (G03) and 2016-2017 (G11). This type of analysis can be made for other geodetic or geophysical phenomena (e.g. Earth rotation parameters or coordinate time series) for selected intervals (sub-daily, daily, weekly, etc.).

The above clock analysis might be helpful, e.g. in determination of uncertainties in positioning and we keep this study for future work. In other words, a correlation analysis can be done between identified noises in Fig. 4 and the Allan deviation curves in the east, north, and up directions. We believe that different noise types of satellite clocks will affect uncertainties in positioning, a similar study can be seen in (Niu et al. 2014; Shi et al. 2019).

\section{Conclusions}

This work shows a method for presenting Allan (and related) variances and the noises associated with it in a simple and accessible way. Moreover, it allows for numerically assigning of the noises type to each pair of $\sigma$ and $\tau$ and shows it as a quantitative and qualitative representation.

\begin{abstract}
Abbreviations
GPS: Global positioning system; GNSS: Global navigation satellite system; ADEV: Allan deviation; AVAR: Allan variance; PM: Phase modulation; FM: Frequency modulation; WPM: White phase modulation; FPM: Flicker phase modulation; WFM: White frequency modulation; FFM: Flicker frequency modulation; RWFM: Random walk frequency modulation; FWFM: Flicker walk frequency modulation; RRFM: Random run frequency modulation; IGS: International GNSS service; MGEX: The Multi-GNSS experiment; MDEV: Modified Allan Deviation; CODE: Centre for Orbit Determination in Europe.
\end{abstract}

\section{Acknowledgements}

Not applicable.

\section{Authors' contributions}

KM 45\%, JK 45\%, IVB 5\%, MB 5\%. All authors read and approved the final manuscript.

\section{Funding}

This paper was made at AGH-UST within statutory research 16.16.150.545 and Initiative for Excellence - Research University grant.

\section{Availability of data and materials}

The datasets analysed during the current study are available in the MGEX repository: http://ftp.aiub.unibe.ch/CODE_MGEX/CODE/2018 (Fig. 3, 4) and http://ftp.aiub.unibe.ch/CODE (Fig. 5).

\section{Competing interests}

Not applicable.

\section{Author details}

${ }^{1}$ Faculty of Mining Surveying and Environmental Engineering, AGH University of Science and Technology, Krakow, Poland. ${ }^{2}$ Faculty of Engineering and Sustainable Development, University of Gävle, 80176 Gävle, Sweden. ${ }^{3}$ Division of Geodesy and Satellite Positioning, Royal Institute of Technology (KTH), 10044 Stockholm, Sweden. ${ }^{4}$ VNIIFTRI, Mendeleevo, Moscow reg, Russian Federation.

Received: 19 November 2020 Accepted: 28 November 2020

Published online: 09 December 2020

\section{References}

Allan DW (1983) Clock characterization tutorial. In: Proceedings of the 15th annual precise time and time interval (PTTI) applications and planning meetingtions and planning meeting. pp 459-475

Allan DW (1987) Time and Frequency (Time-Domain) Characterization, Estimation, and Prediction of Precision Clocks and Oscillators. IEEE Trans Ultrason Ferroelectr Freq Control 34:647-654. https://doi. org/10.1109/T-UFFC.1987.26997

Amiri-Simkooei AR, Tiberius CCJM, Teunissen PJG (2007) Assessment of noise in GPS coordinate time series: Methodology and results. J Geophys Res Solid Earth 112:1-19. https://doi.org/10.1029/2006JB004913

Griggs E, Kursinski ER, Akos D (2014) An investigation of GNSS atomic clock behavior at short time intervals. GPS Solut 18:443-452. https://doi. org/10.1007/s10291-013-0343-7

Heo YJ, Cho J, Heo MB (2010) Improving prediction accuracy of GPS satellite clocks with periodic variation behaviour. Meas Sci Technol. https://doi. org/10.1088/0957-0233/21/7/073001

Hidalgo J, Poulakis P, Köhler J et al (2012) Improving planetary rover attitude estimation via MEMS sensor characterization. Sensors 12:2219-2235. https://doi.org/10.3390/s120202219

Lombardi MA (2016) Evaluating the frequency and time uncertainty of GPS disciplined oscillators and clocks. NCSLI Meas 11:30-44. https://doi. org/10.1080/19315775.2017.1316696

McGrew WF, Zhang X, Fasano RJ et al (2018) Atomic clock performance enabling geodesy below the centimetre level. Nature 564:87-90. https://doi. org/10.1038/s41586-018-0738-2

Niu X, Chen Q, Zhang Q et al (2014) Using Allan variance to analyze the error characteristics of GNSS positioning. GPS Solut 18:231-242. https://doi. org/10.1007/s10291-013-0324-x

Riley WJ (2008) Handbook of frequency stability analysis

Senior KL, Ray JR, Beard RL (2008) Characterization of periodic variations in the GPS satellite clocks. GPS Solut 12:211-225. https://doi.org/10.1007/s1029 $1-008-0089-9$

Shi C, Guo S, Gu S et al (2019) Multi-GNSS satellite clock estimation constrained with oscillator noise model in the existence of data discontinuity. J Geod 93:515-528. https://doi.org/10.1007/s00190-018-1178-3

\section{Publisher's Note}

Springer Nature remains neutral with regard to jurisdictional claims in published maps and institutional affiliations. 